\title{
Dislocation Density by X-ray Diffraction in $\alpha$ Brass Deformed by Rolling and ECAE
}

\author{
Talita Gama Sousa ${ }^{a}$, Saulo Brinco Diniz ${ }^{a}$, André Luiz Pinto ${ }^{b}$, Luiz Paulo Brandao $^{a *}$ \\ ${ }^{a}$ Seção de Engenharia Mecânica e de Materiais, Instituto Militar de Engenharia - IME, \\ Praça General Tibúrcio, 80, Praia Vermelha, CEP 22290-270, Rio de Janeiro, RJ, Brazil \\ ${ }^{b}$ Centro Brasileiro de Pesquisas Físicas - CBPF, Rua Dr. Xavier Sigaud, 150, Urca, \\ CEP 22290-180, Rio de Janeiro, RJ, Brazil
}

Received: December 14, 2014; Revised: August 4, 2015

\begin{abstract}
Dislocations are responsible for most aspects of plastic deformation in metals. In this work, the dislocation density, $\rho$, in brass was estimated after different deformation processes via line broadening of X-ray diffractograms using the Convolutional Multiple Whole Profile Program (CMWP) and by Transmission Electron Microscopy (TEM). In addition, we attempted to evaluate, on a global basis, the influence of crystallographic texture in $\rho$ analysis, making sure that the results obtained by XRD could be trusted even in samples with moderate levels of texture. For this, we compared the $\rho$ values for $\alpha$ brass samples $(66 \% \mathrm{Cu}$ and $34 \% \mathrm{Zn})$ with different levels of texture, one deformed by cold rolling and the other by equal channel angular extrusion (ECAE). The results suggest that using CMWP program it was possible to satisfactorily estimate the dislocation density in $\alpha$ brass. It also the was shown that the results by XRD and by TEM were self-consistent in two samples texturized to different degrees.
\end{abstract}

Keywords: dislocation density, TEM, XRD, a brass, rolling, ECAE

\section{Introduction}

Dislocations are responsible for most aspects of plastic deformation of metals. The dislocation density $(\rho)$ is a microstructural feature extensively studied in the scientific community and can be investigated by several instrumental techniques, such as transmission electron microscopy (TEM) and X-ray diffraction (XRD).

TEM is the oldest and consolidated technique in dislocation density analysis, which can be determined by quantitative stereology support ${ }^{1}$. The estimate $\rho$ through TEM, however, has some disadvantages such as laborious sample preparation and small observation area.

The determination of the dislocation density using XRD is based on the broadening of the diffraction lines. Over the years, methods and programs have been developed to assess efficiently $\rho$ by the X-ray diffraction profile analysis. In this specific research, we used a computer program called CMWP (Convolutional Multiple Whole Profile) developed by a group of researchers at the University Eötvös Loránd ${ }^{2}$. This program describes the diffraction profiles according to various microstructural parameters, including $\rho$. The CMWP follows the idea of Rietveld refinement, i.e., it obtains a theoretical diffraction pattern represented by analytic functions and fits the measured pattern using the mathematical method of least squares ${ }^{2}$.

In this program, the effect of microstrain in peak broadening is caused by dislocations, where the analytic function is represented by a Fourier transform expressed $\mathrm{as}^{2}$ :

*e-mail: brandao@ime.eb.br

$$
A^{D}(L)=\exp \left[-\frac{\pi b^{2}}{2}\left(g^{2} C\right) \rho L^{2} f\left(\frac{L}{R_{e}^{*}}\right)\right]
$$

where $f$ is the strain function, $g$ is the absolute value of the diffraction vector, $L$ is Fourier variable, $b$ is the absolute value of the Burgers vector and $R^{*}$ is a length parameter. The anisotropic effect is considered by the contrast factor of dislocations, $\mathrm{C}$, where through CMWP program it is possible to evaluate either in terms of average contrast factors in case of random oriented samples or in terms of individual factors contrast in case of strongly textured samples ${ }^{3}$.

This study has two parts. Firstly, we compared the estimates of dislocation density using TEM and XRD in annealed and deformed samples. Secondly, we compared the estimates of dislocation density using XRD in deformed samples with moderate and weak texture.

It is known that certain microstructural features, such as texture, can change the diffraction pattern and directly interfere with the results ${ }^{3}$ In order to evaluate the influence of texture on dislocation density results of moderately textured samples, we used two different methods of deformation, the traditional one, cold rolling with $70 \%$ reduction, which induced a moderate texture intensity, and a promising severe deformation process, called ECAE (Equal Channel Angular Extrusion), that induced an even weaker texture for the same degree of deformation ${ }^{4,5}$. Then, the dislocation density values ( $\rho$ ) obtained by XRD was compared for samples resulting from the two deformation methods.

The purpose of this research was to evaluate whether the $\rho$ results could be validated in samples with moderate texture using the average contrast factors rather than individual 
contrast factors, which have to be used for strongly textured materials.

The $\alpha$ brass alloy was chosen for this study because it has a relatively low stacking fault energy that leads to low dynamic recovery, which is an important parameter for evaluating the results of the measurement of dislocation density. The results indicated that even with the presence of moderate texture intensity the $\rho$ estimated by XRD is reliable. They also showed that there was consistency between the TEM and the XRD measures.

\section{Material and Methods}

The $\alpha$-brass alloy ( $66 \% \mathrm{Cu}$ and $34 \% \mathrm{Zn}$ ) supplied by the Paranapanema Company was used as sheets $(3.17 \mathrm{~mm}$ in thickness) for cold rolling and as a round bar $(9.52 \mathrm{~mm}$ in diameter) for ECAE.

The material was annealed (BA) at $600{ }^{\circ} \mathrm{C}$ in air with a soak time of $1 \mathrm{~h}$ and subsequently cooled in the furnace, in order to obtain low dislocation density before deformation.

The samples were subjected to mechanical deformation after pickling by immersion in a $10 \%$ sulfuric acid $\left(\mathrm{H}_{2} \mathrm{SO}_{4}\right)$ solution for $10 \mathrm{~min}$. Specimens were then cold rolled. In the process, the sheets were reduced by the amount of $70 \%$ and $85 \%$ in thickness (B70 and B85, respectively).

For ECAE deformation a circular cross section channel die $10 \mathrm{~mm}$ in diameter and $120^{\circ}$ angle between the equiangular channels was used. Samples were extruded with graphite base lubrication. Specimens were deformed with just two pass denoted as $\mathrm{B} 2 \mathrm{X}$.

In order to analyze the influence of texture in XRD technique, we used cold rolled samples and deformed samples by ECAE, where different stress states were imposed, but with comparable equivalent strain. Thus, the equivalent strain calculated for ECAE, $\bar{\varepsilon}$, is expressed by ${ }^{5}$ :

$$
\bar{\varepsilon}=\frac{2 N}{\sqrt{3}} \cot \varphi
$$

where the angle between the equiangular channel, $\Phi$, is expressed by $2 \varphi$ and $\mathrm{N}$ is the number of passes. Regarding the rolling process (planar deformation) the equivalent true strain, $\bar{\varepsilon}$, is obtained by ${ }^{6}$ :

$$
\bar{\varepsilon}=\frac{2 \sqrt{3}}{3} \varepsilon_{3}
$$

where $\varepsilon_{3}$ is the true thickness strain.

The samples to be analyzed by XRD were mechanically polished with diamond paste and chemically polished with a solution containing 1 part of nitric acid $\left(\mathrm{HNO}_{3}\right), 1 \frac{1}{4}$ parts of acetic acid $\left(\mathrm{CH}_{3} \mathrm{CO}_{2} \mathrm{H}\right)$ and $23 / 4$ parts of phosphoric acid $\left(\mathrm{H}_{3} \mathrm{PO}_{3}\right)^{[7]}$.

The XRD diffractograms were recorded in an X'Pert PRO MRD diffractometer and the values of dislocation density were calculated using the CMWP software.

In this research, the crystallographic texture analyses were performed using the distribution functions (ODF) generated by the popLA code and were obtained from pole figures of the (111), (200) and (220) plans measured by XRD. The cold rolled samples were analyzed in the rolling direction and ECAE deformed samples on the ND-TD (Normal Direction-Transversal Direction) plane.

For the dislocation analysis using TEM performed in a JEOL JEM-2100F, samples were prepared via electrolytic polishing with an electrolytic solution of $30 \%$ nitric acid $\left(\mathrm{HNO}_{3}\right)$ and $70 \%$ methanol $\left(\mathrm{CH}_{3} \mathrm{OH}\right)$ at room temperature.

The dislocation density, obtained by TEM analysis, was determined by the Ham intercept method ${ }^{1}$. This is an intercept counting procedure, where the image magnification is taking into account. In the case of annealed samples, four micrographs were obtained in two different grains; for deformed samples, three micrographs per region were obtained at four different grains ${ }^{8,9}$. The values of sample thickness were obtained by technique of Electron Energy Loss Spectroscopy, EELS.

\section{Results and Discussion}

Figures 1 and 2 show one of several micrographs of the BA and B85 samples, respectively.

Dislocation densities $(\rho)$ obtained by XRD and TEM can be observed in Table 1. Both assessments of dislocation density were consistent for deformed ductile metals and with the sample processing history, providing smaller values for annealed materials than for cold rolled samples ${ }^{10}$. The TEM values were larger than the XRD values (Table 1). When analyzing a region in the TEM, areas where dislocations could be seen might have been preferably selected. Although

Table 1. Dislocation density ( $\rho$ ) obtained through TEM and XRD methods for annealed and cold rolled samples.

\begin{tabular}{ccc}
\hline Samples & $\begin{array}{c}\boldsymbol{\rho}\left(\text { dislocations } / \mathbf{c m}^{2}\right) \\
\text { by TEM }\end{array}$ & $\begin{array}{c}\boldsymbol{\rho}\left(\text { dislocations } / \mathbf{c m}^{2}\right) \\
\text { by XRD }\end{array}$ \\
\hline BA & $3.45 \pm 1.52 \times 10^{9}$ & $9.89 \times 10^{8}$ \\
B85 & $2.70 \pm 0.23 \times 10^{11}$ & $7.58 \times 10^{12}$ \\
\hline
\end{tabular}

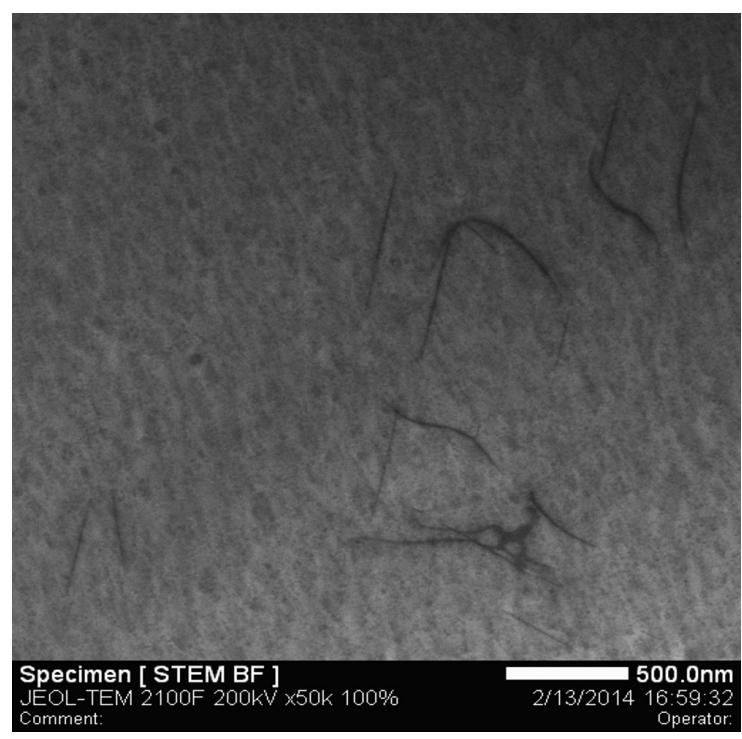

Figure 1. STEM micrograph from BA sample. 
we tried to choose regions as randomly as possible, the need to verify if the contrast was enough to pinpoint dislocations may have led us to look for areas where dislocations were clearly visible. This procedure could have conducted to a systematic error that could have led to overestimate the dislocation density, as already reported.

The opposite was observed for the B85 sample where the values obtained in the XRD analysis were larger than those found for the TEM examination. A potential source of error, in this situation, is the very dense tangle of dislocations observed in the micrographs (Figure 2), that makes it very difficult to count individual dislocations using the intercept method. Undoubtedly, many dislocations were not accounted and included in the $\rho$ calculation, promoting an underestimation of the dislocation density like previously reported by Bailey $\&$ Hirsch $^{9}$. These researchers wrote about attempts to assess dislocation density in areas where $\rho$ were too high and have reported that their estimation was inaccurate. Thus, this agglomerate of high amount of dislocations might have contributed for inaccurate evaluation and could explain the difference between the values for TEM and XRD analysis of B85 samples.

Thus, despite the difference, it can be stated that the values found by both XRD and TEM analysis are appropriate and consistent $^{11,12}$. Although the TEM results were impacted by systematic errors, these could be improved increasing the number of samples and regions analyzed, which is costly and slow. On the other hand, the XDR technique provided reliable and more practical results ${ }^{3}$.

Another significant aim of this work was to evaluate the influence of the crystallographic texture in the dislocation density obtained by XRD. For this purpose, we used cold rolled and ECAE processed $\alpha$ brass, because, as mentioned in the introduction, cold rolling introduces more texture in

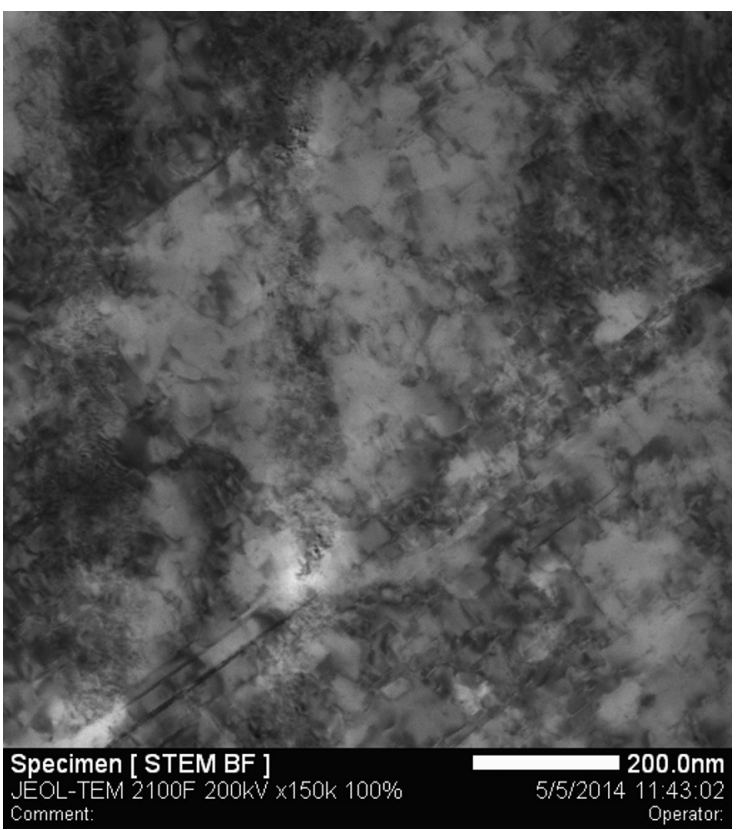

Figure 2. STEM micrograph from B85 sample. a material than ECAE. Also, it is important to notice that B70 sample showed approximately the same equivalent deformation that B2X sample processed by ECAE (1.38 and 1.32 , respectively), thus both samples can exhibit comparable dislocation density measurement (Table 2).

The texture results for the B70 and B2X samples are shown in Figures 3 and 4. In these figures the orientation distribution functions (ODF) follow ROE notation. One can see that sample B70 (Figure 3) shows an intense (7 m.r.o.) brass texture (B), while sample B2X (Figure 4) shows a 3 m.r.o. brass component and a 3 m.r.o.cube (C) component. (Figures 3 and 4)

It can be seen in Table 3 that the dislocation density values obtained for the B70 and B2X samples are of the same order of magnitude, despite the fact that the cold rolled sample has a greater texture level than the sample processed by ECAE. This is taken as evidence that even with presence of moderate texture the the estimates of dislocation density by XRD method using the CMWP program in terms of average contrast factors are reliable in the rolled samples (Table 3).

Table 2. Equivalent strain measures, $\bar{\varepsilon}$.

\begin{tabular}{ccccc}
\hline \multicolumn{3}{c}{ Rolling } & \multicolumn{3}{c}{ ECAE } \\
\hline Reduction & $\varepsilon_{1}$ & $\bar{\varepsilon}$ & N & $\bar{\varepsilon}$ \\
\hline $70 \%$ & 1.20 & 1.38 & 2 & 1.32 \\
\hline
\end{tabular}

Table 3. Dislocation density $(\rho)$ obtained through XRD for the B70 and B2X samples.

\begin{tabular}{cc}
\hline Samples & $\boldsymbol{\rho}\left(\right.$ dislocations $\left./ \mathbf{c m}^{2}\right)$ \\
\hline B70 & $7.48 \times 10^{12}$ \\
B2X & $7.19 \times 10^{12}$ \\
\hline
\end{tabular}

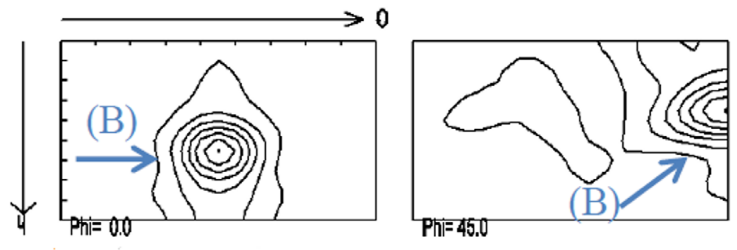

Contours at 122344567

Figure 3. ODF from B70 sample, showing a brass texture component (B).

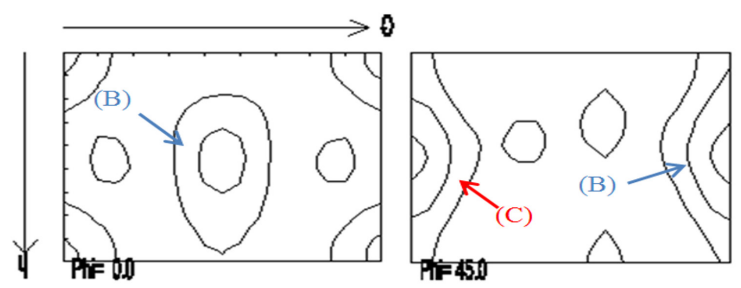

Contorsat 123

Figure 4. ODF from B2X sample, showing cub (C) and brass (B) texture components. 


\section{Conclusion}

From the results obtained in this work, it can be concluded that:

- Dislocation density estimation by the XRD method for a moderately textured sample is reliable and consistent;

- Measurement of dislocation density in moderately textured $\alpha$-brass through stereology measures performed by TEM is qualitatively self-consistent;

- The dislocation density values of deformed samples produced via cold rolling and ECAE showed that the

\section{References}

1. Ham RK. The determination of dislocation densities in thin film. Philosophical Magazine. 1961; 6:1183-1184.

2. Ribárik G. Modeling of diffraction patterns based on microstructural properties. [Thesis]. Budapest: Eötvös Loránd University; 2008.

3. Ribárik $\mathrm{G}$ and Ungár T. Characterization of the microstructure in random and textured polycrystals and single crystals by diffraction line profile analysis. Materials Science and Engineering A. 2010; 528(1):112-121. http://dx.doi.org/10.1016/j.msea.2010.08.059.

4. Furukama M, Horita Z, Nemoto M and Langdon TG. Review: processing of metals by equal-channel angular pressing. Journal of Materials Science. 2001; 36(12):2835-2843. http://dx.doi. org/10.1023/A:1017932417043.

5. Valiev RZ and Langdon TG. Principles of equal-channel angular pressing as a processing tool for grain refinement. Progress in Materials Science. 2006; 51(7):881-981. http:// dx.doi.org/10.1016/j.pmatsci.2006.02.003.

6. Backofen WA. Deformation processing. Boston: AddisonWesley Publishing Company; 1972. presence of moderate texture did not invalidate the measurement of dislocation density, since the results were of the same order of magnitude in both cases.

\section{Acknowledgements}

The authors would like to thank the Brazilian agency CAPES for financial support and Instituto Militar de Engenharia (IME), LABNANO at Centro Brasileiro de Pesquisas Físicas (CBPF) and Universidade Federal de São Carlos (UFSCAR) for the use of laboratory facilities.

7. Vander Voort GF. Metallography: principles and practice. 2nd ed. New York: McGraw-Hill; 1984.

8. Hirsch PB, Howie A, Nicholson RB, Pashley DW and Whelan MJ. Application of the dynamical theory to crystals containing dislocations. In: Hirsch PB, editor. Electron microscopy of thin crystals. 5rd ed. London: Butterworths; 1965. p. 247-275.

9. Bailey JE and Hirsch PB. The dislocation distribution, flow stress, and stored energy in cold-worked polycrystalline silver. Philosophical Magazine. 1960; 5(53):485-497. http://dx.doi. org/10.1080/14786436008238300.

10. Kumar NK, Roy B and Das J. Effect of twin spacing, dislocation density and crystallite size on the strength of nanostructured a-brass. Journal of Alloys and Compounds. 2015; 618:139-145. http://dx.doi.org/10.1016/j.jallcom.2014.08.131.

11. Grabova RB, Gorbatov VK, Ostrovsky ZE and Yuskaev RA. Microstructural study of an irradiated high-nickel alloy by X-ray line profile analysis and TEM observations. Journal of Nuclear Materials. 1997; 240(3):229-235. http://dx.doi. org/10.1016/S0022-3115(96)00678-2.

12. Sousa TG, Diniz SB, Pinto AL, Brandão LP. Dislocantion density evaluation by $\mathrm{X}$-ray diffraction line broadening and TEM of deformed copper. Materials Research. In press. 